\title{
La vieillesse, là où je vais vivre...
}

\section{Jean Martin}

Dr méd., membre de la rédaction

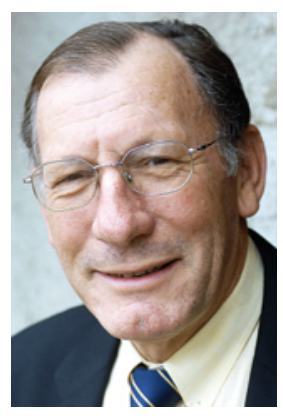

«Je m'intéresse à la vieillesse, parce que c'est là que je vais passer le reste de ma vie» - je paraphrase le comédien américain George Burns, qui parlait du futur («where I'll spend the rest of my life»). Le fait est que, participant à des discussions sur le grand âge, notamment la toujours plus grande quantité - et qualité - de soins que demandent les seniors, je me dis que c'est de moi qu'on parle. Le magazine Time du 26 février 2018 présente un dossier sous le titre "How to live longer better». Extraits: "La mort est vue souvent comme une bonne chose pour une société qui aspire à être créative; si votre temps est compté vous réalisez plus.» «Les personnes âgées sont plus centrées sur le présent.» "Une étude de 2017 a comparé les mots positifs et négatifs dans des blogs de personnes en phase terminale et d'autres qui ne l'étaient pas. Les mourants étaient plus positifs.» Le prof. G.D. Borasio dit que les personnes en fin de vie se montrent plus altruistes.

«Selon le psychologue Erik Erikson, en avançant en âge, ce n'est plus réaliser et amasser des choses qui est le plus important, mais bien les donner plus loin [...] L'idée d'une génération remplaçant l'autre devient un tampon contre l'anxiété.» Il faut apprendre, convenions-nous il y a peu avec un confrère retraité, à prendre le temps de faire - ou de ne pas faire - les choses. Apprendre aussi à accepter l'inachevé (dans notre parcours, quel qu'il soit).

J'assistais récemment à une réunion à ce propos. Un orateur a parlé des vues de C.G Jung sur la dernière partie de la vie.* Il s'agit de retrouver ou développer des choses qu'on a négligées (cela parle à ceux qui ont été pris entièrement ou presque par leur travail durant des décennies). En rapport avec nos réussites d'adultes, de professionnels, il a cité cette formule zen: «Celui qui a atteint son but a manqué tout le reste»!? Ouvrir la porte à la sérénité et trouver du sens, un chemin, au-dedans de soi-même plutôt qu'à l'extérieur quelque chose de nouveau se construit. Redonner du goût à l'attente, a dit quelqu'un. NB: ceci sans pour autant, relève l'orateur, vouloir se débarrasser de sa persona, de son image sociale construite antérieurement.
Le psychiatre Jacques Besson, de Lausanne, a réfléchi sur la verticalité, sur «le divin dans l'homme». Et sur le temps, aux multiples définitions. Etre attentif et saisir le kairos (le moment important et opportun, parfois point de basculement décisif), plutôt que rester fixé sur le chronos (le temps linéaire, celui de la montre). Il parle de Viktor Frankl dont l'expérience en camp de concentration lui a montré que les personnes ayant survécu avaient une vie intérieure leur permettant de trouver du sens. Aussi, Frankl a développé la logothérapie (narratologie): comme les témoignages de soignants à cette réunion, des travaux en nombre croissant illustrent que la narratologie (leur permettre de parler) est d'un grand bénéfice pour les personnes âgées - pour (re) trouver et (re)donner sens.

Ne pas croire, bien sûr, qu'il y a une seule doctrine juste en ces matières. Les idées changent (au gré d'acquis scientifiques aussi). Une gérontologue-théologienne a relevé qu'au milieu du $\mathrm{XX}^{\mathrm{e}}$ siècle avait été développé, à propos des vieux, le modèle de l'activité (à maintenir), puis celui du "successful aging» (en s'efforçant de prévenir, de s'adapter et de s'autonomiser). Aujourd'hui elle suggère de retrouver la noblesse de la notion d'accomplissement. Comme dernière étape de l'âge qui avance, elle a évoqué celle de la résilience, comprise comme l'apprentissage à croître à travers les déclins (les déficits, les déchirures). Citant aussi le sociologue F. Höpflinger, de Zurich: «Nous avons appris à rester jeunes plus longtemps, nous n'avons pas appris à aimer la vieillesse."

Croître à travers les déclins, aimer la vieillesse ... Idéalement, c'est à quoi nous devons nous préparer. Ce beau programme toutefois sera-t-il celui adopté par le plus grand nombre? La tentation pourra être de sauter cette ultime phase - qui peut être sereine, "accomplie», mais dont certains penseront qu'on peut s'en passer, l'éviter par un raccourci... Tel que le suicide assisté, dont je pense qu'il doit rester une transgression mais dont on voit qu'il fait de moins en moins peur. Il y a quelques mois, un vieil ami m’a demandé d'être à côté de lui dans ces circonstances - bien particulières. 